\title{
A VARIANT OF THE PRIME NUMBER THEOREM
}

\author{
KUI LIU, JIE WU \& ZHISHAN YANG
}

Abstract. Let $\Lambda(n)$ be the von Mangoldt function, and let $[t]$ be the integral part of real number $t$. In this note, we prove that for any $\varepsilon>0$ the asymptotic formula

$$
\sum_{n \leqslant x} \Lambda\left(\left[\frac{x}{n}\right]\right)=x \sum_{d \geqslant 1} \frac{\Lambda(d)}{d(d+1)}+O_{\varepsilon}\left(x^{9 / 19+\varepsilon}\right) \quad(x \rightarrow \infty)
$$

holds. This improves a recent result of Bordellès, which requires $\frac{97}{203}$ in place of $\frac{9}{19}$.

\section{INTRODUCTION}

The prime number theorem is a basic result in number theory and has many applications. Denoting by $\Lambda(n)$ the von Mangoldt function, the prime number theorem states, in strong form, as follows: there is a constant $c>0$ such that for $x \rightarrow \infty$, we have

$$
\sum_{n \leqslant x} \Lambda(n)=x+O\left(x \exp \left\{-c(\log x)^{3 / 5}(\log \log x)^{-1 / 5}\right\}\right)
$$

and

$$
\sum_{n \leqslant x} \Lambda(n)=x+O_{\varepsilon}\left(x^{1 / 2+\varepsilon}\right) \Leftrightarrow \text { Riemann Hypothesis, }
$$

where $\varepsilon$ is an arbitrarily small positive constant. Clearly it is also interesting to study the distribution of prime numbers in different sequences of integers such as the arithmetic progressions, the Beatty sequence $([\alpha n+\beta])_{n \geqslant 1}$, the Piatetski-Shapiro sequence $\left(\left[n^{c}\right]\right)_{n \geqslant 1}$, etc, where $[t]$ denotes the integral part of the real number. For example, Banks and Shparlinski [2, Corollary 5.6] proved the following result: Let $\alpha$ and $\beta$ be fixed real numbers with $\alpha>0$, irrational and of finite type, then there is a positive constant $c=c(\alpha, \beta)$ such that

$$
\sum_{n \leqslant x} \Lambda([\alpha n+\beta])=x+O\left(x \exp \left\{-c(\log x)^{3 / 5}(\log \log x)^{-1 / 5}\right\}\right)
$$

as $x \rightarrow \infty$. About works related to the Piatetski-Shapiro prime number theorem, we infer the reader to see [12, 8, 13]. On the other hand, Bordellès-Dai-Heyman-Pan-Shparlinski [4] established an asymptotic formula of

$$
S_{f}(x):=\sum_{n \leqslant x} f\left(\left[\frac{x}{n}\right]\right)
$$

under some simple assumptions of $f$. Subsequently, Wu [16] and Zhai [17] improveed their results independently. In particular, applying [16, Theorem 1.2(i)] or [17, Theorem 1] to the von Mangoldt function $\Lambda(n)$, we have

$$
S_{\Lambda}(x)=x \sum_{d \geqslant 1} \frac{\Lambda(d)}{d(d+1)}+O_{\varepsilon}\left(x^{1 / 2+\varepsilon}\right)
$$

Date: May 25, 2021.

2010 Mathematics Subject Classification. 11N37, 11 L07.

Key words and phrases. The prime number theorem, Exponential sums, Vaughan's identity. 
for $x \geqslant 1$. With the help of the Vaughan identity and the method of one-dimensional exponential sum, Ma and $\mathrm{Wu}$ [11] breaked the $\frac{1}{2}$-barrier:

$$
S_{\Lambda}(x)=x \sum_{d \geqslant 1} \frac{\Lambda(d)}{d(d+1)}+O_{\varepsilon}\left(x^{35 / 71+\varepsilon}\right) \quad(x \geqslant 1) .
$$

Very recently Bordellès [3, Corollary 1.3] sharpened the exponent $\frac{35}{71}$ to $\frac{97}{203}$ by using a result of Baker on 2-dimensional exponential sums [1, Theorem 6].

The aim of this short note is to propose a better exponent by establishing an estimate on 3-dimensional exponential sums (see Proposition 3.1 below).

Theorem 1. For any $\varepsilon>0$, we have

$$
S_{\Lambda}(x)=x \sum_{d \geqslant 1} \frac{\Lambda(d)}{d(d+1)}+O_{\varepsilon}\left(x^{9 / 19+\varepsilon}\right) \quad \text { as } x \rightarrow \infty .
$$

For comparaison, we have $\frac{97}{203} \approx 04778$ and $\frac{9}{19} \approx 0.4736$. In fact, Bordellès established a more general result (see [3, Theorem 1.1]):

$$
S_{\Lambda}(x)=x \sum_{d \geqslant 1} \frac{\Lambda(d)}{d(d+1)}+O_{\varepsilon}\left(x^{\frac{14(\kappa+1)}{29 \kappa-\lambda+30}+\varepsilon}\right) \quad(x \rightarrow \infty),
$$

where $(\kappa, \lambda)$ is an exponent pair satisfying $\kappa \leqslant \frac{1}{6}$ and $\lambda^{2}+\lambda+3-\kappa(5+9 \kappa-\lambda)>0$. The exponent $\frac{97}{203}$ comes from the choice of $(\kappa, \lambda)=\left(\frac{13}{84}+\varepsilon, \frac{55}{84}+\varepsilon\right)$ - Bourgain's new exponent pair [5, Theorem 6]. We note that under the exponent pair hypothesis (i.e. $\left(\varepsilon, \frac{1}{2}+\varepsilon\right)$ is an exponent pair, see [7]), Bordellès' (1.3) only gives the exponent $\frac{28}{59} \approx 0.4745$, which is larger than our constant $\frac{9}{19} \approx 0.4736$.

Some related works on the quantity (1.1) can be found in [10, 15].

\section{Preliminary lemmas}

In this section, we shall cite three lemmas, which will be needed in the next section. The first one is [․, Lemma 1].

Lemma 2.1. Let $\alpha \in \mathbb{R}^{*}$ and $\beta \in \mathbb{R}^{*}$. For $M \geqslant 1, N \geqslant 1$ and $\Delta>0$, define

$$
\mathcal{D}(M, N ; \Delta):=\left|\left\{(m, \tilde{m}, n, \tilde{n}) \in \mathbb{N}^{4}: m, \tilde{m} \sim M ; n, \tilde{n} \sim N ;\left|\left(\frac{m}{\tilde{m}}\right)^{\alpha}-\left(\frac{n}{\tilde{n}}\right)^{\beta}\right| \leqslant \Delta\right\}\right|,
$$

where $m \sim M$ means that $M<m \leqslant 2 M$. Then we have

$$
\mathcal{D}(M, N) \ll_{\alpha, \beta} M N \log (2 M N)+\Delta(M N)^{2}
$$

uniformly for $M \geqslant 1, N \geqslant 1$ and $\Delta>0$, where the implied constant depends on $\alpha$ and $\beta$.

The second one is the Vaughan identity [14, formula (3)].

Lemma 2.2. There are six real arithmetical functions $\alpha_{k}(n)$ verifying $\left|\alpha_{k}(n)\right| \ll_{\varepsilon} n^{\varepsilon}$ for $(n \geqslant 1,1 \leqslant k \leqslant 6)$ such that, for all $D \geqslant 100$ and any arithmetical function $g$, we have

$$
\sum_{D<d \leqslant 2 D} \Lambda(d) g(d)=S_{1}+S_{2}+S_{3}+S_{4},
$$


where

$$
\begin{aligned}
S_{1} & :=\sum_{m \leqslant D^{1 / 3}} \alpha_{1}(m) \sum_{D<m n \leqslant 2 D} g(m n), \\
S_{2} & :=\sum_{m \leqslant D^{1 / 3}} \alpha_{2}(m) \sum_{D<m n \leqslant 2 D} g(m n) \log n, \\
S_{3} & :=\sum_{\substack{D^{1 / 3}<m, n \leqslant D^{2 / 3} \\
D<m n \leqslant 2 D}} \alpha_{3}(m) \alpha_{4}(n) g(m n), \\
S_{4} & :=\sum_{\substack{1 / 3 \\
D^{1 / 3}<m, n \leqslant D^{2 / 3} \\
D<m n \leqslant 2 D}} \alpha_{5}(m) \alpha_{6}(n) g(m n) .
\end{aligned}
$$

The sums $S_{1}$ and $S_{2}$ are called as type $I, S_{3}$ and $S_{4}$ are called as type II.

The third one is due to Vaaler (see [7, Theorem A.6]).

Lemma 2.3. Let $\psi(t):=\{t\}-\frac{1}{2}$, where $\{t\}$ means the fractional part of real number $t$. For $x \geqslant 1$ and $H \geqslant 1$, we have

$$
\psi(x)=-\sum_{1 \leqslant|h| \leqslant H} \Phi\left(\frac{h}{H+1}\right) \frac{\mathrm{e}(h x)}{2 \pi \mathrm{i} h}+R_{H}(x),
$$

where $\mathrm{e}(t):=\mathrm{e}^{2 \pi \mathrm{i} t}, \Phi(t):=\pi t(1-|t|) \cot (\pi t)+|t|$ and the error term $R_{H}(x)$ satisfies

$$
\left|R_{H}(x)\right| \leqslant \frac{1}{2 H+2} \sum_{0 \leqslant|h| \leqslant H}\left(1-\frac{|h|}{H+1}\right) \mathrm{e}(h x) .
$$

\section{Multiple exponential Sums}

Let $\alpha>0, \beta>0, \gamma>0$ and $\delta \in \mathbb{R}$ be some constants. For $X>0, H \geqslant 1, M \geqslant 1$ and $N \geqslant 1$, define

$$
S_{\delta}=S_{\delta}(H, M, N):=\sum_{h \sim H} \sum_{m \sim M} \sum_{n \sim N} a_{h, m} b_{n} \mathrm{e}\left(X \frac{M^{\beta} N^{\gamma}}{H^{\alpha}} \frac{h^{\alpha}}{m^{\beta} n^{\gamma}+\delta}\right),
$$

where the $a_{h, m}$ and $b_{n}$ are complex numbers such that $\left|a_{h, m}\right| \leqslant 1$ and $\left|b_{n}\right| \leqslant 1$. When $\delta=0$, this sum has been studied by Heath-Brown [8] and Fouvry-Iwaniec [6]. The aim of this section is to prove an estimate of $S_{\delta}$ by adapting and refining Heath-Brown's approach (see also [9]). The following proposition will play a key role in the proof of Theorem 1.

Proposition 3.1. Under the previous notation, for any $\varepsilon>0$ we have

$$
\begin{aligned}
S_{\delta} & \ll\left((X H M N)^{1 / 2}+(H M)^{1 / 2} N+H M N^{1 / 2}+X^{-1 / 2} H M N\right) X^{\varepsilon}, \\
S_{\delta} & \ll\left(\left(X^{\kappa} H^{2+\kappa} M^{2+\kappa} N^{1+\kappa+\lambda}\right)^{1 /(2+2 \kappa)}+H M N^{1 / 2}\right. \\
& \left.+(H M)^{1 / 2} N+X^{-1 / 2} H M N\right) X^{\varepsilon}
\end{aligned}
$$

uniformly for $M \geqslant 1, N \geqslant 1, H \leqslant M^{\beta-1} N^{\gamma}$ and $|\delta| \leqslant 1 / \varepsilon$, where $(\kappa, \lambda)$ is an exponent pair and the implied constant depends on $(\alpha, \beta, \gamma, \varepsilon)$ only.

Proof. Obviously we have

$$
h^{\alpha} m^{-\beta} \leqslant C_{1} H^{\alpha} M^{-\beta}=: \Xi \quad(h \sim H, m \sim M),
$$


where the $C_{j}=C_{j}(\alpha, \beta, \gamma)$ denotes some positive constant depending on $(\alpha, \beta, \gamma)$ at most. Let $K \geqslant 1$ be a parameter to be chosen later. Introducing the following set

$$
T_{k}:=\left\{(h, m): h \sim H, m \sim M, \Xi(k-1)<h^{\alpha} m^{-\beta} K \leqslant \Xi k\right\},
$$

we can write

$$
S_{\delta}=\sum_{n \sim N} b_{n} \sum_{k \leqslant K} \sum_{(h, m) \in T_{k}} a_{h, m} \mathrm{e}\left(X \frac{M^{\beta} N^{\gamma}}{H^{\alpha}} \frac{h^{\alpha}}{m^{\beta} n^{\gamma}+\delta}\right) .
$$

By the Cauchy-Schwarz inequality, we derive

$$
\left|S_{\delta}\right|^{2} \leqslant N K \sum_{n \sim N} \sum_{k \leqslant K} \sum_{(h, m) \in T_{k}} \sum_{\left(h^{\prime}, m^{\prime}\right) \in T_{k}} a_{h, m} \overline{a_{h^{\prime}, m^{\prime}}} \mathrm{e}\left(X \frac{M^{\beta} N^{\gamma}}{H^{\alpha}}\left(\frac{h^{\alpha}}{m^{\beta} n^{\gamma}+\delta}-\frac{h^{\prime \alpha}}{m^{\prime \beta} n^{\gamma}+\delta}\right)\right) .
$$

Inverting the order of summations, it follows that

$$
\left|S_{\delta}\right|^{2} \leqslant N K \sum_{k \leqslant K} \sum_{(h, m) \in T_{k}} \sum_{\left(h^{\prime}, m^{\prime}\right) \in T_{k}}\left|\mathcal{S}\left(h, h^{\prime}, m, m^{\prime}\right)\right|,
$$

where

$$
\mathcal{S}\left(h, h^{\prime}, m, m^{\prime}\right):=\sum_{n \sim N} \mathrm{e}\left(X \frac{M^{\beta} N^{\gamma}}{H^{\alpha}}\left(\frac{h^{\alpha}}{m^{\beta} n^{\gamma}+\delta}-\frac{h^{\prime \alpha}}{m^{\prime \beta} n^{\gamma}+\delta}\right)\right) .
$$

Noticing that $(h, m),\left(h^{\prime}, m^{\prime}\right) \in T_{k}$ imply $\left|h^{\alpha} m^{-\beta}-h^{\prime \alpha} m^{-\beta}\right| \leqslant \Xi K^{-1}$, we can write

$$
\left|S_{\delta}\right|^{2} \leqslant N K \sum_{\substack{h, h^{\prime} \sim H \\\left|h^{\alpha} m^{-\beta}-h^{\prime \alpha} m^{\prime-\beta}\right| \leqslant \Xi K^{-1}}}\left|\mathcal{S}\left(h, h^{\prime}, m, m^{\prime}\right)\right| \leqslant N K\left(S_{\delta}^{\dagger}+S_{\delta}^{\sharp}\right),
$$

where

$$
\begin{aligned}
& S_{\delta}^{\dagger}:=\sum_{\substack{h, h^{\prime} \sim H \\
m, m^{\prime} \sim M \\
\mid h^{\alpha} m^{-\beta}-h^{\prime \alpha} m^{\prime-\beta} \sim=(H M)^{-1}}}\left|\mathcal{S}\left(h, h^{\prime}, m, m^{\prime}\right)\right|, \\
& \left|h^{\alpha} m^{-\beta}-h^{\prime \alpha} m^{\prime-\beta}\right| \leqslant \Xi(H M)^{-1} \\
& S_{\delta}^{\sharp}:=\quad \sum_{h, h^{\prime} \sim H} \sum_{m, m^{\prime} \sim M} \quad\left|\mathcal{S}\left(h, h^{\prime}, m, m^{\prime}\right)\right| \text {. } \\
& \Xi(H M)^{-1}<\left|h^{\alpha} m^{-\beta}-h^{\prime \alpha} m^{\prime-\beta}\right| \leqslant \Xi K^{-1}
\end{aligned}
$$

[We have made the convention that $S_{\delta}^{\sharp}=0$ if $K \leqslant H M$.] Since

$$
\left|\frac{h^{\alpha}}{m^{\beta}}-\frac{h^{\prime \alpha}}{m^{\prime \beta}}\right| \leqslant \frac{\Xi}{H M} \Rightarrow\left|\frac{h^{\alpha}}{h^{\prime \alpha}}-\frac{m^{\beta}}{m^{\prime \beta}}\right| \leqslant \frac{C_{2}}{\Xi}\left|\frac{h^{\alpha}}{m^{\beta}}-\frac{h^{\prime \alpha}}{m^{\prime \beta}}\right| \leqslant \frac{C_{2}}{H M},
$$

Lemma 2.1 implies that the number of $\left(h, h^{\prime}, m, m^{\prime}\right)$ verifying $\left|h^{\alpha} m^{-\beta}-h^{\prime \alpha} m^{\prime-\beta}\right| \leqslant$ $\Xi(H M)^{-1}$ is $\ll \mathcal{D}\left(H, M ; C_{2} / H M\right) \ll_{\varepsilon} H M X^{\varepsilon}$. Thus we have trivially

$$
S_{\delta}^{\dagger} \ll_{\varepsilon} H M N X^{\varepsilon} .
$$

Next we bound $S_{\delta}^{\sharp}$. We write

$$
\begin{aligned}
& \frac{h^{\alpha}}{m^{\beta} n^{\gamma}+\delta}-\frac{h^{\prime \alpha}}{m^{\prime \beta} n^{\gamma}+\delta} \\
& =\frac{1}{n^{\gamma}}\left(\frac{h^{\alpha}}{m^{\beta}}-\frac{h^{\prime \alpha}}{m^{\prime \beta}}\right)-\frac{\delta}{n^{\gamma}}\left(\frac{h^{\alpha}}{m^{\beta}\left(m^{\beta} n^{\gamma}+\delta\right)}-\frac{h^{\prime \alpha}}{m^{\prime \beta}\left(m^{\prime \beta} n^{\gamma}+\delta\right)}\right)=: f(n) .
\end{aligned}
$$

Since $H \leqslant M^{\beta-1} N^{\gamma}$, we have

$$
\left|\frac{h^{\alpha}}{m^{\beta}\left(m^{\beta} n^{\gamma}+\delta\right)}-\frac{h^{\prime \alpha}}{m^{\prime \beta}\left(m^{\prime \beta} n^{\gamma}+\delta\right)}\right| \leqslant \frac{C_{3} \Xi}{M^{\beta} N^{\gamma}} \ll \frac{\Xi}{H M} \quad\left(h, h^{\prime} \sim H ; m, m^{\prime} \sim M\right),
$$


Therefore for $\left(h, h^{\prime}, m, m^{\prime}\right)$ verifying $\Xi(H M)^{-1}<\left|h^{\alpha} m^{-\beta}-h^{\prime \alpha} m^{\prime-\beta}\right| \leqslant \Xi K^{-1}$, the first member on the right-hand side of (3.8) dominates the second one. Split $\left(\Xi(H M)^{-1}, \Xi K^{-1}\right]$ into dyadic intervals $(\Delta \Xi, 2 \Delta \Xi]$ with $1 / H M \leqslant \Delta \leqslant 1 / K$. Take $K=\max \left\{2 C_{4} X / N, 1\right\}$ such that for $\left(h, h^{\prime}, m, m^{\prime}\right)$ verifying $\Xi \Delta<\left|h^{\alpha} m^{-\beta}-h^{\prime \alpha} m^{\prime-\beta}\right| \leqslant 2 \Xi \Delta$ we have

$$
\max _{n \sim N}\left|f^{\prime}(n)\right|=C_{4} X \Delta N^{-1} \leqslant \frac{1}{2} \text {. }
$$

By Kusmin-Landau's inequality and Lemma 2.1, we have

$$
S_{\delta}^{\sharp} \ll_{\varepsilon} X^{\varepsilon} \max _{4 / H M \leqslant \Delta \leqslant 1 / K} \Delta(H M)^{2}\left(X \Delta N^{-1}\right)^{-1} \ll_{\varepsilon} X^{-1+\varepsilon}(H M)^{2} N .
$$

Combining (3.7) and (3.9) with (3.6) gives us

$$
\begin{aligned}
\left|S_{\delta}\right|^{2} & \ll_{\varepsilon}\left(H M N^{2} K+X^{-1}(H M N)^{2} K\right) X^{\varepsilon} \\
& \ll_{\varepsilon}\left(X H M N+H M N^{2}+(H M)^{2} N+X^{-1}(H M N)^{2}\right) X^{\varepsilon},
\end{aligned}
$$

which is equivalent to (3.2).

Next we prove (3.4). If $X \leqslant H M$, then (3.2) implies that

$$
S_{\delta} \ll_{\varepsilon}\left((H M)^{1 / 2} N+H M N^{1 / 2}+X^{-1 / 2} H M N\right) X^{\varepsilon} .
$$

Now we can suppose that $X \geqslant H M$. Applying the exponent pair $(\kappa, \lambda)$ to $\mathcal{S}\left(h, h^{\prime}, m, m^{\prime}\right)$. and using Lemma 2.1, we can derive that

$$
\begin{aligned}
S_{\delta}^{\sharp} & \ll_{\varepsilon} X^{\varepsilon} \max _{4 / H M \leqslant \Delta \leqslant 1 / K} \Delta(H M)^{2}\left(\left(X \Delta N^{-1}\right)^{\kappa} N^{\lambda}+\left(X \Delta N^{-1}\right)^{-1}\right) \\
& \ll_{\varepsilon} X^{\varepsilon}\left(X^{\kappa} H^{2} M^{2} N^{-\kappa+\lambda} K^{-1-\kappa}+X^{-1} H^{2} M^{2} N\right) .
\end{aligned}
$$

Combining (3.7) and (3.11) with (3.6) gives us

$$
\left|S_{\delta}\right|^{2} \ll_{\varepsilon}\left(X^{\kappa} H^{2} M^{2} N^{1-\kappa+\lambda} K^{-\kappa}+H M N^{2} K\right) X^{\varepsilon}
$$

for all $K \in[1, H M]$, where we have removed the term $X^{-1} H^{2} M^{2} N^{2} K\left(\leqslant H M N^{2} K\right.$ since we have suppose that $X \geqslant H M)$. Noticing that this estimate is trivial if $K \geqslant H M$, we can optimise the parameter $K$ over $[1, \infty)$ to get

$$
\left|S_{\delta}\right|^{2} \ll_{\varepsilon}\left(\left(X^{\kappa} H^{2+\kappa} M^{2+\kappa} N^{1+\kappa+\lambda}\right)^{1 /(1+\kappa)}+H M N^{2}\right) X^{\varepsilon}
$$

Combining this with (3.10), we obtain (3.4).

\section{A KEY INEQUALity}

The aim of this section is to prove the following proposition, which will play a key role for the proof of Theorem 1. Define

$$
\mathfrak{S}_{\delta}(x, D):=\sum_{d \sim D} \Lambda(d) \psi\left(\frac{x}{d+\delta}\right) .
$$

Proposition 4.1. Let $\delta \notin-\mathbb{N}$ be a fixed constant. Then we have

$$
\begin{aligned}
\mathfrak{S}_{\delta}(x, D) \ll & \left(\left(x^{2 \kappa} D^{3+\lambda}\right)^{1 /(4 \kappa+4)}+D^{5 / 6}\right. \\
& \left.+\left(x^{3 \kappa^{\prime}} D^{-2 \kappa^{\prime}+2 \lambda^{\prime}+1}\right)^{1 /\left(3 \kappa^{\prime}+3\right)}+\left(x^{3 \kappa^{\prime}} D^{-5 \kappa^{\prime}+2 \lambda^{\prime}+1}\right)^{1 / 3}\right) x^{\varepsilon} .
\end{aligned}
$$

uniformly for $x \geqslant 3$ and $1 \leqslant D \leqslant x^{2 / 3}$, where $(\kappa, \lambda)$ and $\left(\kappa^{\prime}, \lambda^{\prime}\right)$ are exponent pairs. In particular, uniformly for $x^{6 / 13} \leqslant D \leqslant x^{2 / 3}$ we have

$$
\mathfrak{S}_{\delta}(x, D) \ll_{\varepsilon}\left(x^{2} D^{7}\right)^{1 / 12} x^{\varepsilon} .
$$


Proof. We apply the Vaughan identity (2.1) with $g(d)=\psi\left(\frac{x}{d+\delta}\right)$ to write

$$
\mathfrak{S}_{\delta}(x, D)=\mathfrak{S}_{\delta, 1}+\mathfrak{S}_{\delta, 2}+\mathfrak{S}_{\delta, 3}+\mathfrak{S}_{\delta, 4}
$$

where

$$
\begin{aligned}
\mathfrak{S}_{\delta, 1} & :=\sum_{m \leqslant D^{1 / 3}} \alpha_{1}(m) \sum_{D<m n \leqslant 2 D} \psi\left(\frac{x}{m n+\delta}\right), \\
\mathfrak{S}_{\delta, 2} & :=\sum_{m \leqslant D^{1 / 3}} \alpha_{2}(m) \sum_{D<m n \leqslant 2 D} \psi\left(\frac{x}{m n+\delta}\right) \log n, \\
\mathfrak{S}_{\delta, 3}: & \sum_{\substack{D^{1 / 3}<m, n \leqslant D^{2 / 3} \\
D<m n \leqslant 2 D}} \alpha_{3}(m) \alpha_{4}(n) \psi\left(\frac{x}{m n+\delta}\right), \\
\mathfrak{S}_{\delta, 4}: & \sum_{\substack{D^{1 / 3}<m, n \leqslant D^{2 / 3} \\
D<m n \leqslant 2 D}} \alpha_{5}(m) \alpha_{6}(n) \psi\left(\frac{x}{m n+\delta}\right) .
\end{aligned}
$$

Firstly we estimate $\mathfrak{S}_{\delta, 3}$. In view of Lemma 2.3 , we can write

$$
\mathfrak{S}_{\delta, 3}=-\frac{1}{2 \pi \mathrm{i}} \sum_{H^{\prime}} \sum_{M} \sum_{N}\left(\mathfrak{S}_{\delta, 3}^{b}\left(H^{\prime}, M, N\right)+\overline{\mathfrak{S}_{\delta, 3}^{b}}\left(H^{\prime}, M, N\right)\right)+\sum_{M} \sum_{N} \mathfrak{S}_{\delta, 3}^{\dagger}(M, N) .
$$

where $M N \asymp D$ (i.e. $D \ll M N \ll D), \alpha(h):=\frac{H^{\prime}}{h} \Phi\left(\frac{h}{H+1}\right) \ll 1$ and

$$
\begin{aligned}
& \mathfrak{S}_{\delta, 3}^{b}\left(H^{\prime}, M, N\right):=\frac{1}{H^{\prime}} \sum_{\substack { h \sim H^{\prime} \\
\begin{subarray}{c}{m \sim M \\
D<m n \leqslant 2 D{ h \sim H ^ { \prime } \\
\begin{subarray} { c } { m \sim M \\
D < m n \leqslant 2 D } }\end{subarray}} \sum_{\substack{n \sim N \\
\text { a }}} \alpha(h) \alpha_{3}(m) \alpha_{4}(n) \mathrm{e}\left(\frac{h x}{m n+\delta}\right), \\
& \mathfrak{S}_{\delta, 3}^{\dagger}(M, N):=\sum_{\substack{m \sim M \\
D<m n \leqslant 2 D}} \sum_{\substack{n \sim N \\
\text { a }}} \alpha_{3}(m) \alpha_{4}(n) R_{H}\left(\frac{x}{m n+\delta}\right) .
\end{aligned}
$$

Firstly we bound $\mathfrak{S}_{\delta, 3}^{b}\left(H^{\prime}, M, N\right)$. We remove the extra multiplicative condition $D<$ $m n \leqslant 2 D$ at the cost of a factor $\log x$. On the other hand, in view of the symmetry of the variables $m$ and $n$, we can suppose that

$$
D^{1 / 3} \leqslant M \leqslant D^{1 / 2} \leqslant N \leqslant D^{2 / 3} .
$$

By Proposition 3.1 with $\alpha=\beta=\gamma=1$ and $(X, H, M, N)=\left(x H^{\prime} / M N, H^{\prime}, M, N\right)$, we have

$$
\mathfrak{S}_{\delta, 3}^{b}\left(H^{\prime}, M, N\right) \ll_{\varepsilon}\left(\left(x^{\kappa} M^{2} N^{1+\lambda}\right)^{1 /(2+2 \kappa)}+M N^{1 / 2}+M^{1 / 2} N+\left(x^{-1} D H^{\prime}\right)^{1 / 2}\right) x^{\varepsilon},
$$

provided $H^{\prime} \leqslant H \leqslant N$.

Secondly we bound $\mathfrak{S}_{\delta, 3}^{\dagger}(M, N)$. Using (2.2), we have

$$
\begin{aligned}
\mathfrak{S}_{\delta, 3}^{\dagger}(M, N) & \ll x^{\varepsilon} \sum_{m \sim M} \sum_{n \sim N}\left|R_{H}\left(\frac{x}{m n+\delta}\right)\right| \\
& \ll \frac{x^{\varepsilon}}{H} \sum_{m \sim M} \sum_{n \sim N} \sum_{0 \leqslant|h| \leqslant H}\left(1-\frac{|h|}{H+1}\right) \mathrm{e}\left(\frac{h x}{m n+\delta}\right) \\
& \ll x^{\varepsilon}\left(D H^{-1}+\max _{1 \leqslant H^{\prime} \leqslant H}\left|\widetilde{\mathfrak{S}}_{\delta, 3}^{\dagger}\left(H^{\prime}, M, N\right)\right|\right),
\end{aligned}
$$

where

$$
\widetilde{\mathfrak{S}}_{\delta, 3}^{\dagger}\left(H^{\prime}, M, N\right):=\frac{1}{H} \sum_{m \sim M} \sum_{n \sim N} \sum_{h \sim H^{\prime}}\left(1-\frac{|h|}{H+1}\right) \mathrm{e}\left(\frac{h x}{m n+\delta}\right) .
$$


Clearly we can bound $\widetilde{\mathfrak{S}}_{\delta, 3}^{\dagger}\left(H^{\prime}, M, N\right)$ in the same way as $\mathfrak{S}_{\delta, 3}^{b}\left(H^{\prime}, M, N\right)$ and obtain

$$
\begin{aligned}
\mathfrak{S}_{\delta, 3}^{\dagger}(M, N) \ll_{\varepsilon} & \left(D H^{-1}+\left(x^{\kappa} M^{2} N^{1+\lambda}\right)^{1 /(2+2 \kappa)}\right. \\
& \left.+M N^{1 / 2}+M^{1 / 2} N+\left(x^{-1} D H\right)^{1 / 2}\right) x^{\varepsilon},
\end{aligned}
$$

provided $H \leqslant N$. Combining (4.7) and (4.8) with (4.5) and using (4.6), we find that

$$
\begin{aligned}
\mathfrak{S}_{\delta, 3} & \ll_{\varepsilon}\left(D H^{-1}+\left(x^{\kappa} M^{2} N^{1+\lambda}\right)^{1 /(2+2 \kappa)}+M N^{1 / 2}+M^{1 / 2} N+\left(x^{-1} D H\right)^{1 / 2}\right) x^{\varepsilon} \\
& \ll_{\varepsilon}\left(D H^{-1}+\left(x^{2 \kappa} D^{3+\lambda}\right)^{1 /(4+4 \kappa)}+D^{5 / 6}+\left(x^{-1} D H\right)^{1 / 2}\right) x^{\varepsilon},
\end{aligned}
$$

provided $H \leqslant D^{1 / 2}(\leqslant N)$. Optimising $H$ over $\left[1, D^{1 / 2}\right]$, it follows that

$$
\mathfrak{S}_{\delta, 3} \ll_{\varepsilon}\left(\left(x^{2 \kappa} D^{3+\lambda}\right)^{1 /(4+4 \kappa)}+D^{5 / 6}+\left(x^{-1} D^{2}\right)^{1 / 3}\right) x^{\varepsilon} .
$$

Clearly the same estimate also holds for $\mathfrak{S}_{\delta, 4}$.

On the other hand, let $\left(\kappa^{\prime}, \lambda^{\prime}\right)$ be an exponent pair, then [11, (5.16)] gives us

$$
\mathfrak{S}_{\delta, j} \ll\left(\left(x^{3 \kappa^{\prime}} D^{-2 \kappa^{\prime}+2 \lambda^{\prime}+1}\right)^{1 /\left(3 \kappa^{\prime}+3\right)}+x^{\kappa^{\prime}} D^{\left(-5 \kappa^{\prime}+2 \lambda^{\prime}+1\right) / 3}+x^{-1} D^{2}\right) x^{\varepsilon} .
$$

for $j=1,2$. Inserting (4.10) and (4.9) into (4.4) and using the fact that

$$
\max \left\{\left(x^{-1} D^{2}\right)^{1 / 3}, x^{-1} D^{2}\right\} \leqslant D^{5 / 6}
$$

for $1 \leqslant D \leqslant x^{2 / 3}$, we get (4.2).

Taking $(\kappa, \lambda)=\left(\kappa^{\prime}, \lambda^{\prime}\right)=\left(\frac{1}{2}, \frac{1}{2}\right)$ in (4.2), we find that

$$
\mathfrak{S}_{\delta}(x, D) \ll\left(\left(x^{2} D^{7}\right)^{1 / 12}+D^{5 / 6}+\left(x^{3} D^{2}\right)^{1 / 9}+\left(x^{3} D^{-1}\right)^{1 / 6}\right) x^{\varepsilon},
$$

which implies (4.3), since the last three terms can be absorbed by the first one provided $x^{6 / 13} \leqslant D \leqslant x^{2 / 3}$.

\section{Proof of Theorem 1}

Let $N \in\left[x^{6 / 13}, x^{1 / 2}\right)$ be a parameter which can be chosen later. First we write

$$
\sum_{n \leqslant x} \Lambda\left(\left[\frac{x}{n}\right]\right):=S_{1}(x)+S_{2}(x)
$$

with

$$
S_{1}(x):=\sum_{n \leqslant N} \Lambda\left(\left[\frac{x}{n}\right]\right), \quad S_{2}(x):=\sum_{N<n \leqslant x} \Lambda\left(\left[\frac{x}{n}\right]\right) .
$$

We have trivially

$$
S_{1}(x) \ll_{\varepsilon} N x^{\varepsilon} .
$$

Next we bound $S_{2}(x)$. Putting $d=[x / n]$, then $x / n-1<d \leqslant x / n \Leftrightarrow x /(d+1)<n \leqslant x / d$. Thus we can write

$$
\begin{aligned}
S_{2}(x) & =\sum_{d \leqslant x / N} \Lambda(d) \sum_{x /(d+1)<n \leqslant x / d} 1 \\
& =\sum_{d \leqslant x / N} \Lambda(d)\left(\frac{x}{d}-\psi\left(\frac{x}{d}\right)-\frac{x}{d+1}+\psi\left(\frac{x}{d+1}\right)\right) \\
& =x \sum_{d \geqslant 1} \frac{\Lambda(d)}{d(d+1)}+\mathcal{R}_{1}(x)-\mathcal{R}_{0}(x)+O(N),
\end{aligned}
$$


where we have used the following bounds

$$
x \sum_{d>x / N} \frac{\Lambda(d)}{d(d+1)} \ll_{\varepsilon} N x^{\varepsilon}, \quad \sum_{d \leqslant N} \Lambda(d)\left(\psi\left(\frac{x}{d+1}\right)-\psi\left(\frac{x}{d}\right)\right) \ll_{\varepsilon} N x^{\varepsilon}
$$

and defined

$$
\mathcal{R}_{\delta}(x)=\sum_{N<d \leqslant x / N} \Lambda(d) \psi\left(\frac{x}{d+\delta}\right) .
$$

Writing $D_{j}:=x /\left(2^{j} N\right)$, we have $x^{6 / 13} \leqslant N \leqslant D_{j} \leqslant x / N \leqslant x^{7 / 13}$ for $0 \leqslant j \leqslant \frac{\log \left(x / N^{2}\right)}{\log 2}$ since $x^{6 / 13} \leqslant N \leqslant x^{1 / 2}$. Thus we can apply (4.3) of Proposition 4.1 to get

$$
\begin{aligned}
\left|\mathcal{R}_{\delta}(x)\right| & \leqslant \sum_{0 \leqslant j \leqslant \log \left(x / N^{2}\right) / \log 2}\left|\mathfrak{S}_{\delta}\left(x, D_{j}\right)\right| \\
& \ll \sum_{0 \leqslant j \leqslant \log \left(x / N^{2}\right) / \log 2}\left(x^{2} D_{j}^{7}\right)^{1 / 12} x^{\varepsilon} \\
& \ll\left(x^{9} N^{-7}\right)^{1 / 12} x^{\varepsilon} .
\end{aligned}
$$

Putting this into (5.3) and taking $N=x^{9 / 19}$, we find that

$$
S_{2}(x)=x \sum_{d \geqslant 1} \frac{\Lambda(d)}{d(d+1)}+O_{\varepsilon}\left(x^{9 / 19+\varepsilon}\right) .
$$

Inserting (5.2) with $N=x^{9 / 19}$ and (5.4) into (5.1), we get the required result.

Acknowledgement. This work is supported in part by the National Natural Science Foundation of China (Grant Nos. 12071238, 11771252, 11971370 and 12071375) and by the NSF of Chongqing (Grant No. cstc2019jcyj-msxm1651).

\section{REFERENCES}

[1] R. C. Baker, Sums of two relatively prime cubes, Acta Arith. 129 (2007), 103-146.

[2] William D. Banks and Igor E. Shparlinski, Prime numbers with Beatty sequences, Colloquium Math. 115 (2009), No. 2, 147-157.

[3] O. Bordellès, On certain sums of number theory, arXiv:2009.05751v2 [math.NT] 25 Nov 2020.

[4] O. Bordellès, L. Dai, R. Heyman, H. Pan and I. E. Shparlinski, On a sum involving the Euler function, J. Number Theory 202 (2019), 278-297.

[5] J. Bourgain, Decoupling, expoential sums and the Riemann zeta function, J. Amer. Math. Soc. 30 (2017), 205-224.

[6] E. Fouvry and H. Iwaniec, Exponential sums with monomials, J. Number Theory 33 (1989), 331333.

[7] S. W. Graham and G. Kolesnik, Van der Corput's Method of Exponential Sums, Cambridge University Press, Cambridge, 1991.

[8] D. R. Heath-Brown, The Pjateckǐ̌-S̆apiro Prime Number Theorem, J. Number Theory 16 (1983), $242-266$.

[9] H.-Q. Liu \& J. Wu, Numbers with a large prime factor, Acta Arith. 89 (1999), 163-187.

[10] J. Ma \& H.-Y. Sun, On a sum involving the divisor function, Period. Math. Hung. , to appear.

[11] J. Ma \& J. Wu, On a sum involving the von Mangoldt function, Period. Math. Hung. 83 (2021), Number 1, 39-48.

[12] I. I. Pjateckiü-S̆apiro, On the distribution of prime numbers in sequences of the form $[f(n)]$, Mat. Sb. 33 (1953), 559-566.

[13] J. Rivat \& J. Wu, Prime numbers of the forms [ $n^{c}$, Glasg. Math. 43 (2001), no. 2, 237-254.

[14] R. C. Vaughan, An elementary method in prime number theory, Acta Arith. 37 (1980), 111-115.

[15] J. Wu, On a sum involving the Euler totient function, Indag. Mathematicae 30 (2019), 536-541. 
[16] J. Wu, Note on a paper by Bordellès, Dai, Heyman, Pan and Shparlinski, Period. Math. Hung. 80 (2020), Number 1, 95-102.

[17] W. Zhai, On a sum involving the Euler function, J. Number Theory 211 (2020), 199-219.

Kui Liu, School of Mathematics and Statistics, Qingdao University, 308 Ningxia Road, Qingdao, Shandong 266071, China

Email address: liukui@qdu.edu.cn

Jie Wu, CNRS UMR 8050, Laboratoire D'Analyse et de Mathématiques Appliquées, Université Paris-Est Créteil, 94010 Créteil cedex, France

Email address: jie.wu@u-pec.fr

Zhishan Yang, School of Mathematics and Statistics, Qingdao University, 308 Ningxia Road, Qingdao, Shandong 266071, China

Email address: zsyang@qdu.edu.cn 